Journal of Engineering and Applied Sciences 7 (6): 389-394, 2012

ISSN: $1816-949 \mathrm{X}$

(C) Medwell Journals, 2012

\title{
Numerical and Experimental Simulation of Flow Pattern in Sudden and Gradual Canal Expansions
}

\author{
${ }^{1} \mathrm{~J}$. Mamizadeh and ${ }^{2}$ S.A. Ayyoubzadeh \\ ${ }^{1}$ Ilam University, Iran \\ ${ }^{2}$ Tarbiat Modares University, Iran
}

\begin{abstract}
In order to examine the flow pattern in canal with sudden and gradual expansion, FLUENT software is used. In this case, 3-dimensional simulations are conducted. The turbulence model is used to simulate turbulence. Desired canal with coarse, medium and fine mesh size is selected for simulation. The results in canal with sudden expansion showed that the vortex formed by coarse mesh size and number of iteration $>400$ is a symmetric and for small and medium mesh size is symmetric. In order to analyze experimentally and numerically more closely, the pattern of flow in a flume with sudden and gradual expansion is investigated. Results showed that in $11.5^{\circ}$ angle, the pattern of asymmetry in length and width of the canal is negligible but in $23.5^{\circ}$ angle like the sudden expansion, the flow pattern is asymmetric. The asymmetrical flow pattern can be viewed with some minor changes such as roughness coefficient in river or reservoir geometry.
\end{abstract}

Key words: Flow pattern, symmetric flow, sudden expansion, geometry, Iran

\section{INTRODUCTION}

Circular flow is a major of flow problems in hydraulics science which due to narrowing or expansion in the canals, presence of obstacles in the path of the canal or bridges piers and other factors arise. For example, sudden expansion of a canal cause significant pressure and velocity drop and generated vortex depending on depth, flow rate and expansion. Many experimenters have been observed that the flow in symmetric expansions is asymmetric. Some of these situations are documented in Graber $(1982,2006)$ who gave an explanation of such phenomenon. He provided a predictive method that agreed well with the experimental observations and then extended it theoretically to analyze proposed corrective measures. Asymmetric flow patterns may occur in perfectly symmetric abrupt expansions in which the main flow deflects and attaches arbitrarily to one wall of the expansion. The adverse effects include lengthening of the distance to full-width flow in expansions, inefficient operation of screens in screen canals and increasing the potential for vortexing in pump intakes. Cherdron et al. (1978) studied the detailed description of the velocity characteristics of the asymmetric flows which form in symmetric, 2-dimensional, plane, sudden-expansion geometries by flow visualization and laser-doppler anemometry. The flow and geometry boundary conditions which give rise to asymmetric flow are indicated and the reason for the phenomenon is shown to lie in disturbances generated at the edge of the expansion and amplified in the shear layers. Zhou (1995) develop a depth average mathematical model and test it in a canal with sudden expansion. Result showed that the flow rate, flow lines and vortex. Formed on both sides are asymmetric. Aloui and Souhar (2000) presents an experimental study of recirculating flows downstream of a flat duct sudden expansion. Results of measurements of average and RMS quantities of the pressure $(\mathrm{P})$ and the axial velocity $(\mathrm{U})$ exhibit the asymmetry of the flow behind the sudden expansion as in the 2-dimensional case. Escudier et al. (2002) reported an experimental investigation of turbulent flow through a plane sudden expansion. Not only was the mean flow found to be strongly asymmetric but integration of the mean axial velocity profiles revealed significant departures from two dimensionality along the centerplane of the expansion duct. The numerical analysis of the turbulent fluid flow through an axi-symmetric sudden expansion passage has been carried out by Roy et al. (2010).

The recirculation bubble generated due to the sudden expansion of the passage is observed to reduce in size and strength with the increase in the reynolds number. But the size and strength of the recirculation bubble increases with the increase in the expansion ratio. Theoretical studies have been made by Roul and Dash (2011) to determine the pressure drops caused by abrupt

Corresponding Author: J. Mamizadeh, Ilam University, Iran 
flow area expansion/contraction in small circular pipes for two-phase flow of air and water mixtures at room temperature and near atmospheric pressure. The pressure drop is determined by extrapolating the computed pressure profiles upstream and downstream of the expansion/contraction. Based on the numerical results as well as experimental data, correlations are developed for two-phase flow pressure drops caused by the flow area contraction as well as expansion. The review research indicates that few studies done on flow pattern in sudden and gradual expansions and therefore evaluated in this study. In this research, flow pattern in sudden and gradual expansions studied with laboratory work and CFD simulation model.

\section{MATERIALS AND METHODS}

FLUENT is a Computational Fluid Dynamics (CFD) software package to simulate fluid flow problems. It uses the finite-volume method to solve the governing equations for a fluid. It provides the capability to use different physical models such as incompressible or compressible, inviscid or viscous, laminar or turbulent, etc. Geometry and grid generation is done using GAMBIT which is the preprocessor bundled with FLUENT. This software uses a control-volume-based technique to convert a general scalar transport equation to an algebraic equation that can be solved numerically. Pressurevelocity coupling is achieved by using five algorithms SIMPLE, SIMPLEC, PISO, Coupled and Fractional step. In the case of incompressible turbulent flow and averaged in time, conservation of mass 1 and momentum conservation 2 expressed as:

$$
\begin{gathered}
\frac{\partial \overline{\mathrm{u}}_{\mathrm{i}}}{\partial \mathrm{x}_{\mathrm{i}}}=0 \\
\frac{\partial \overline{\mathrm{u}}_{\mathrm{i}}}{\partial \mathrm{t}}+\overline{\mathrm{u}}_{\mathrm{j}} \frac{\partial \overline{\mathrm{u}}_{\mathrm{i}}}{\partial \mathrm{x}_{\mathrm{j}}}=-\frac{1}{\rho} \frac{\partial \overline{\mathrm{p}}}{\partial \mathrm{x}_{\mathrm{i}}}+ \\
\mathrm{g}_{\mathrm{i}}+\frac{\partial}{\partial \mathrm{x}_{\mathrm{j}}}\left(\nu \frac{\partial \overline{\mathrm{u}}_{\mathrm{i}}}{\partial \mathrm{x}_{\mathrm{j}}}-\mathrm{u}_{\mathrm{i}}^{\prime} \mathrm{u}_{\mathrm{j}}^{\prime}\right)
\end{gathered}
$$

Where:

$$
\begin{aligned}
\mathrm{u}_{\mathrm{i}} & =\text { Velocity component in } \mathrm{x}_{\mathrm{i}} \text { direction } \\
v & =\text { Kinematic viscosity } \\
\rho & =\text { Density } \\
\mathrm{g}_{\mathrm{i}} & =\text { Gravity acceleration component in } \mathrm{x}_{\mathrm{i}} \text { direction } \\
\mathrm{p} & =\text { Pressure } \\
-\mathrm{u}_{\mathrm{i}}^{\prime} \mathbf{u}_{\mathrm{j}}^{\prime} & =\text { Reynolds stress }
\end{aligned}
$$

This equation contains four main components of the unknown velocity in three directions and pressure. Also includes six components of unknown Reynolds stress equation. Thus, system of equations is not closed and turbulence model must be used. Turbulence model is used in this study. In the other part of research, flow pattern in sudden and gradual expansions studied with laboratory work.

\section{RESULTS AND DISCUSSION}

Simulated flow pattern in sudden expansion: This part of research, compare the result of flow pattern in a sudden expansion $\left(90^{\circ}\right.$ angle from centerline of canal) canal with FLUENT Model and developed mathematical model by Zhou (1995). The entrance canal is $1.4 \mathrm{~m}$ wide and $14 \mathrm{~m}$ long and the expanded canal is $2.8 \mathrm{~m}$ wide and $14 \mathrm{~m}$ long. There is no bed slope in the canal. The upstream entrance velocities are; $u=0.63 \mathrm{~m} \mathrm{sec}^{-1}, v=0$ and the flow depth $\mathrm{y}=0.2 \mathrm{~m}$ as the downstream boundary condition is considered (Fig. 1).

In accordance to Fig. 1, the velocity inlet along with inlet turbulence intensity and hydraulic depth are the upstream boundary condition and outflow is the downstream boundary condition. Boundary condition for bottom and side wall of canal is wall. Symmetry boundary condition is used for top of the canal. Hydraulic flow field details are shown in Table 1.

Turbulent and under critical flow is established in canal. Due to the turbulent flow, turbulence models have been used. SIMPLE algorithm for coupling the velocity field and pressure is used.

Desired canal with coarse, medium and fine mesh size is selected for simulation. Coarse and medium mesh size are; $\Delta \mathrm{x}=\Delta \mathrm{y}=0.28 \mathrm{~m}$ and $\Delta \mathrm{x}=\Delta \mathrm{y}=0.14 \mathrm{~m}$. Fine mesh size was carried out in compliance with first node spacing criteria from the wall. The first node spacing is calculated for $\mathrm{k}-\varepsilon$ and RSM equal to $0.00136 \mathrm{~m}$. Figure 2 shows the three types of coarse, medium and fine mesh size for problem solving.

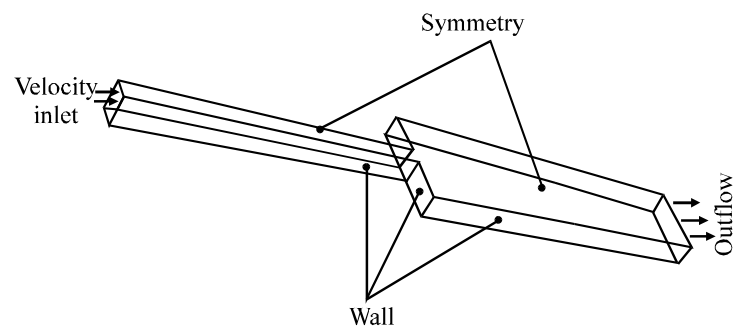

Fig. 1: Canal for simulation (Zhou, 1995)

Table 1: Hydraulic properties of canal

\begin{tabular}{lccccc}
\hline $\begin{array}{l}\text { Turbulence } \\
\text { intensity }\end{array}$ & $\begin{array}{l}\text { Hydraulic } \\
\text { depth }(\mathrm{m})\end{array}$ & $\mathrm{Re}$ & $\mathrm{Fr}$ & $\mathrm{V}\left(\mathrm{m} \mathrm{sec}^{-1}\right)$ & $\mathrm{Q}\left(\mathrm{m}^{3} \mathrm{sec}^{-1}\right)$ \\
\hline 0.03 & 0.2 & 126000 & 0.45 & 0.63 & 0.1764 \\
\hline
\end{tabular}



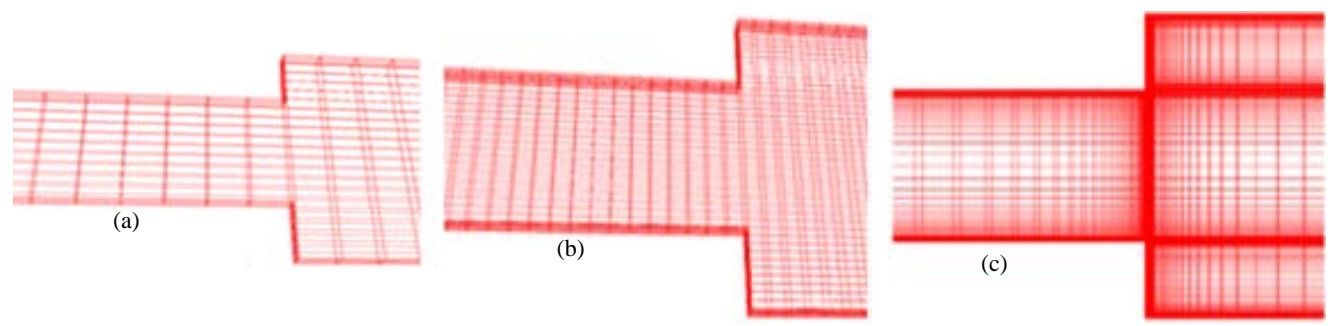

Fig. 2: a) Coarse mesh: 9726 node; b) Medium mesh: 68651 node; c) Fine mesh: 270291 node
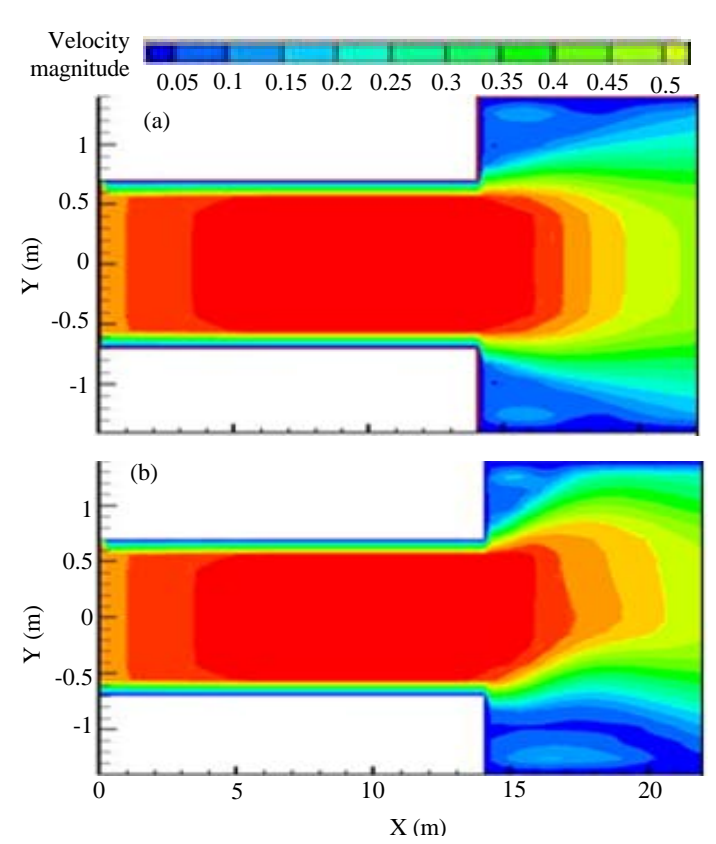

Fig. 3: Flow pattern in canal. Coarse mesh size: a) After 400 interation; b) After 2000 interation

Figure 3 showed the results of the coarse grid model after 400 and 2000 iteration. Flow pattern formed after 400 iteration in Fig. 3a consists of two symmetrical vortex on both sides expansion and a high flow velocity in the central line will be gradually slowed down in $\mathrm{x}$ direction. Figure 3b showed that after 2000 iteration, the flow diverted to one side and an asymmetric flow pattern occurred. Asymmetric flow pattern in this case are due to coarse mesh and also added errors for higher iterations. It should be noted that these results is similar to Zhou (1995) results.

Figure 4 showed the results of model for the medium and fine mesh. Flow pattern consists of two symmetrical vortex on both sides expansion and a high flow velocity in the central line will be gradually slowed down in $\mathrm{x}$ direction.

Figure 5 showed comparing the results of model for coarse, medium and fine mesh. Longitudinal and transverse axis represents the canal width and velocity

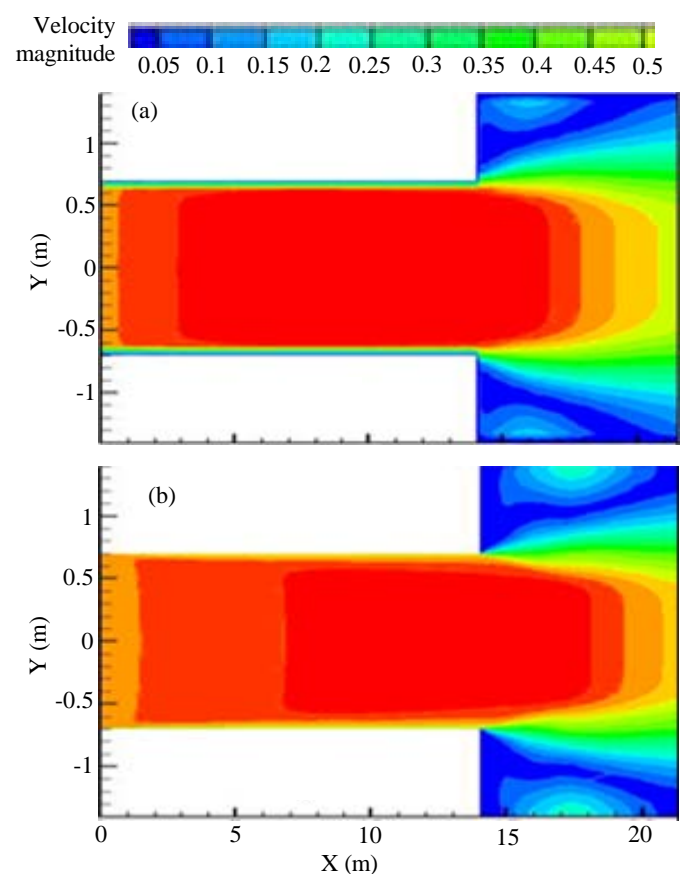

Fig. 4: Flow pattern in canal: a) Medium mesh size; b) Fine mesh size

magnitude respectively. During the $10 \mathrm{~m}$ of canal (Fig. 5a). Results do not differ much but in sudden expansion place for example $14.5 \mathrm{~m}$ (Fig. 5b) coarse and medium mesh size do differ much because of wall function criteria and the accuracy of their answers is slightly low. Also, coarse mesh size showed the higher velocity magnitude in the centerline of canal rather than medium and fine mesh size.

\section{Flow pattern simulation in laboratory flume with sudden} expansion: In order to examine more closely the flow pattern in the sudden expansion a laboratory flume is constructed and a series of experiments were performed on it. Then, flume is studied with fluent numerical model. The flume consists of two sections. Section 1 (River section) have $5 \mathrm{~m}$ length, $16 \mathrm{~cm}$ width, $30 \mathrm{~cm}$ depth and no bed slope. Section 2 (reservoir section) have $10 \mathrm{~m}$ length, $96 \mathrm{~cm}$ width and longitudinal slope $2 \%$. At the end of flume a weir located with $45 \mathrm{~cm}$ elevation. Hydraulic flow 

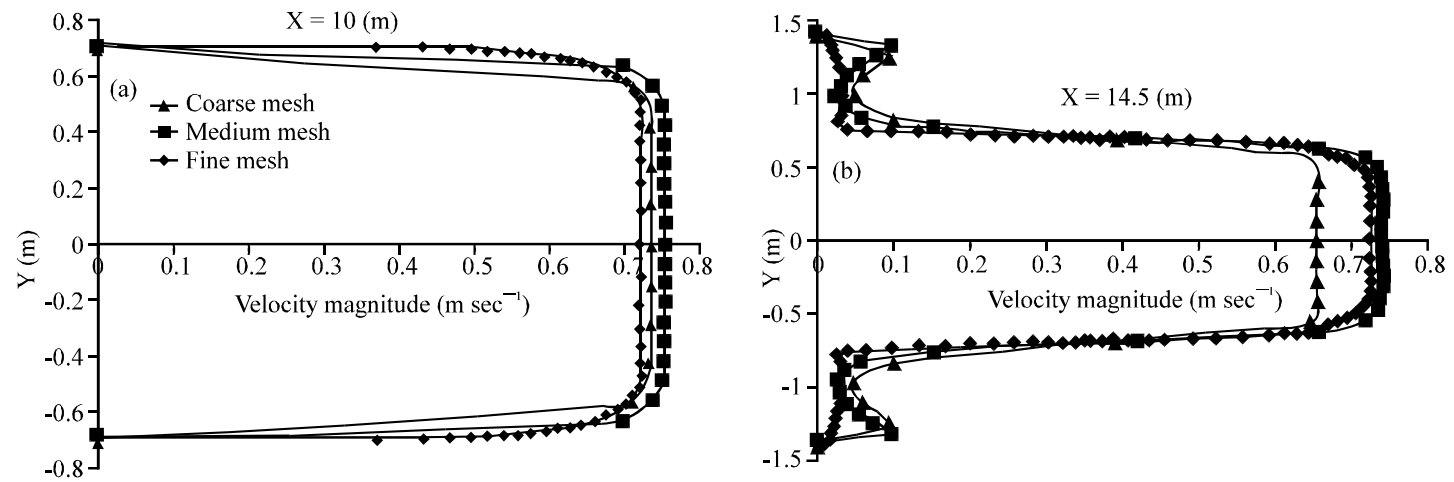

Fig. 5: Result for simulation

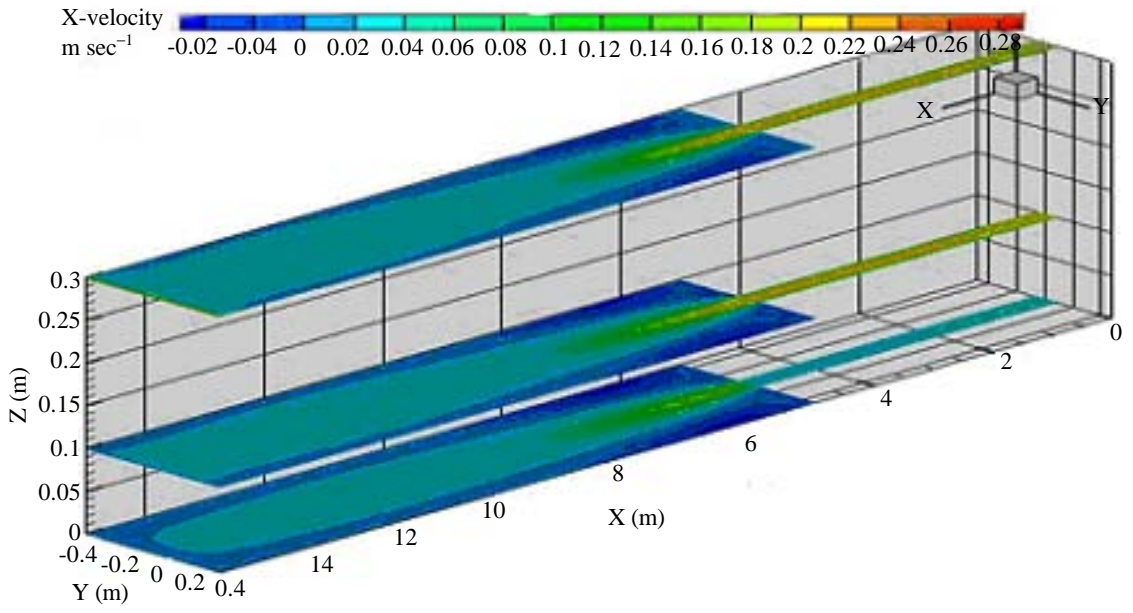

Fig. 6: $\mathrm{x}$-velocity in flume

Table 2: Hydraulic properties of flume

\begin{tabular}{lccccc}
$\begin{array}{l}\text { Turbulence } \\
\text { intensity }\end{array}$ & $\begin{array}{c}\text { Hydraulic } \\
\text { depth }(\mathrm{m})\end{array}$ & $\mathrm{Re}$ & $\mathrm{Fr}$ & $\mathrm{V}\left(\mathrm{m} \mathrm{sec}^{-1}\right)$ & $\mathrm{Q}\left(\mathrm{litsec}^{-1}\right)$ \\
\hline 0.03 & 0.3 & 28125 & 0.055 & 0.094 & 4.5 \\
0.03 & 0.3 & 56250 & 0.110 & 0.178 & 9.0 \\
\hline
\end{tabular}

field details shown in Table 2. Turbulent and under critical flow in a canal is established. Due to the turbulent flow $\mathrm{k}-\varepsilon$ and RSM turbulence model is used. SIMPLE algorithm for coupling the velocity field and pressure is used.

In this case, input velocity perpendicular to the boundary is used for upstream boundary condition and outflow for downstream boundary condition. In order to calculate the flow field, turbulence intensity and hydraulic diameter in upstream 0.3 and $5 \%$ is applied, respectively. Wall boundary condition for the walls and the symmetry boundary condition for top of the canal are applied. According to the first node calculation ( $4 \mathrm{~mm}$ from bottom of canal) mesh near the wall are small and growth $20 \%$ in two adjacent cells farther from wall. Figure 6 shows the result of turbulence model in which flow field are symmetrical at different levels of depth, longitudinal and transverse direction.
Simulated flow pattern simulation with non-uniform roughness: Flow pattern formed in the laboratory flume with sudden expansion using the numerical model was symmetrical and was asymmetric with the experimental observations. In the other hand, numerical model and experimental results did not match together. So in this part, non-uniformity of roughness on the flow pattern is studied. In order to evaluate the effect of non-uniform wall roughness on the flow field, the roughness coefficient of flume in the left part is selected $50 \%$ higher than left part of the flume. Figure 7 and 8 show the streamline in the surface and lower layer of flow with same roughness coefficient and roughness increase in left part. In the case of same roughness, river flows into the reservoir completely symmetric while for the change of the roughness is deflected to the right. Increased roughness of the canal and reservoir, cause more resistance against the flow and the resulting flow is deflected to the right part.

Flow pattern simulation in laboratory flume with gradual expansion: The purpose of this part, numerical and 

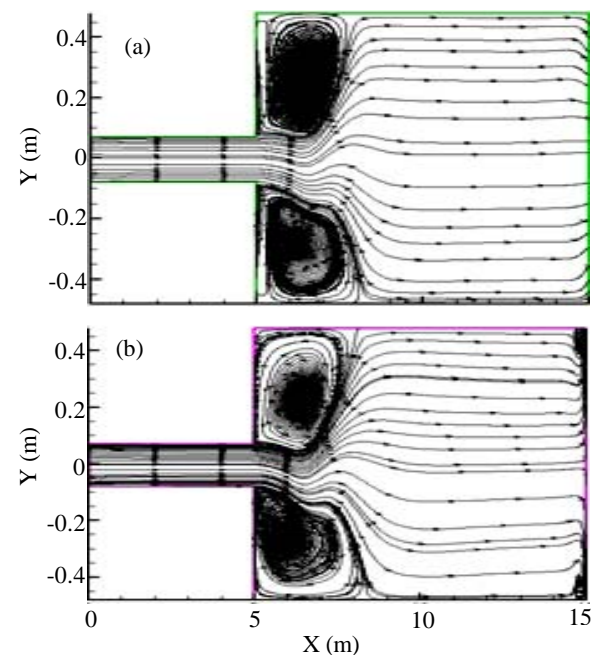

Fig. 7: Stream line for uniform roughness: a) Upper layer; b) Lower layer
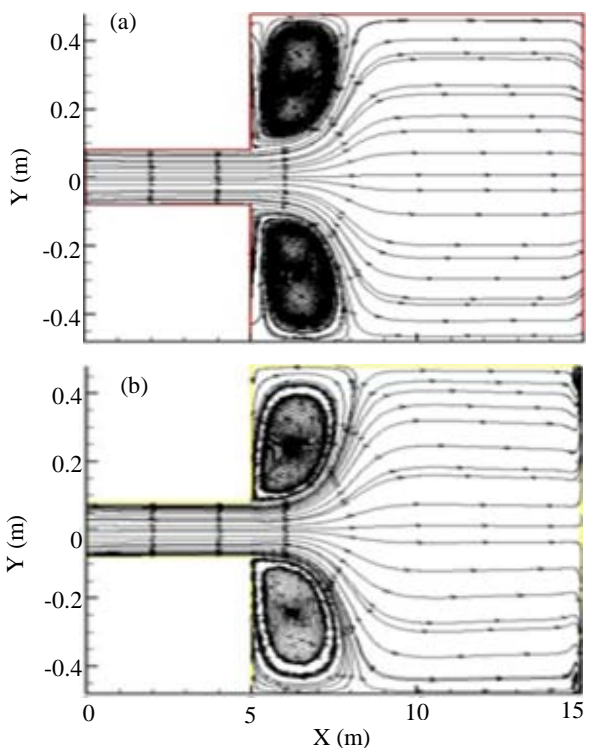

Fig. 8: Stream line for non-uniform roughness: a) Upper layer; b) Lower layer

experimental study of flow pattern in the gradual expansion with 11.5 and $23.5^{\circ}$ angles from the central line of the canal. For numerical simulation, hydraulic flow field is similar to sudden expansion. Figure 9 shows the streamlines in the surface layer for gradual expansion with 11.5 and $23.5^{\circ}$ angles. Result showed that streamlines in river and reservoir in the surface layer is symmetric.

Also, series of experiment are conducted to study hydraulic flow in this case. Figure 10 and 11 compared the result of numerical and experimental longitudinal velocity within the $\mathrm{x}$ and $\mathrm{y}$ direction for two gradual angles expansion, respectively. According to Fig. 10 and 11, the velocity magnitude within the numerical model for

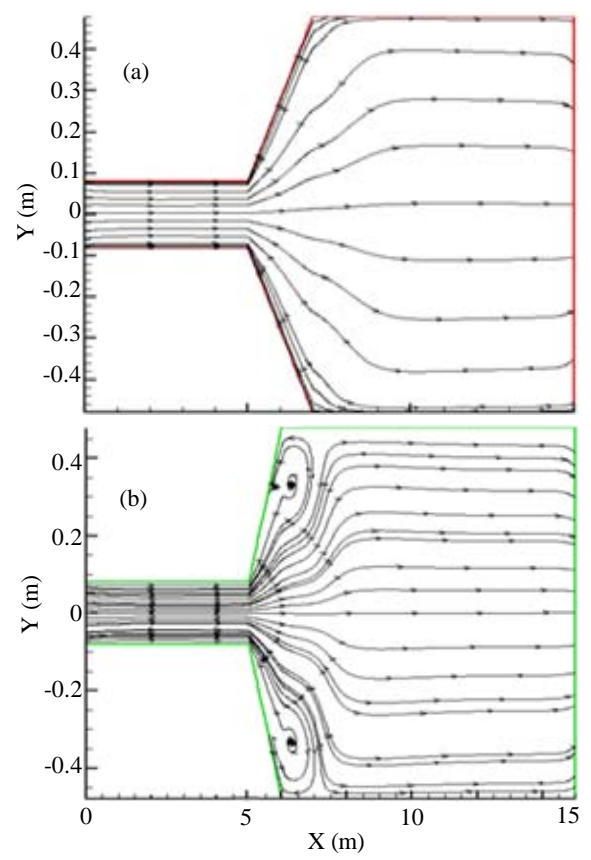

Fig. 9: Stream line foe surface layer: a) $11.5^{\circ}$; b) $23.5^{\circ}$
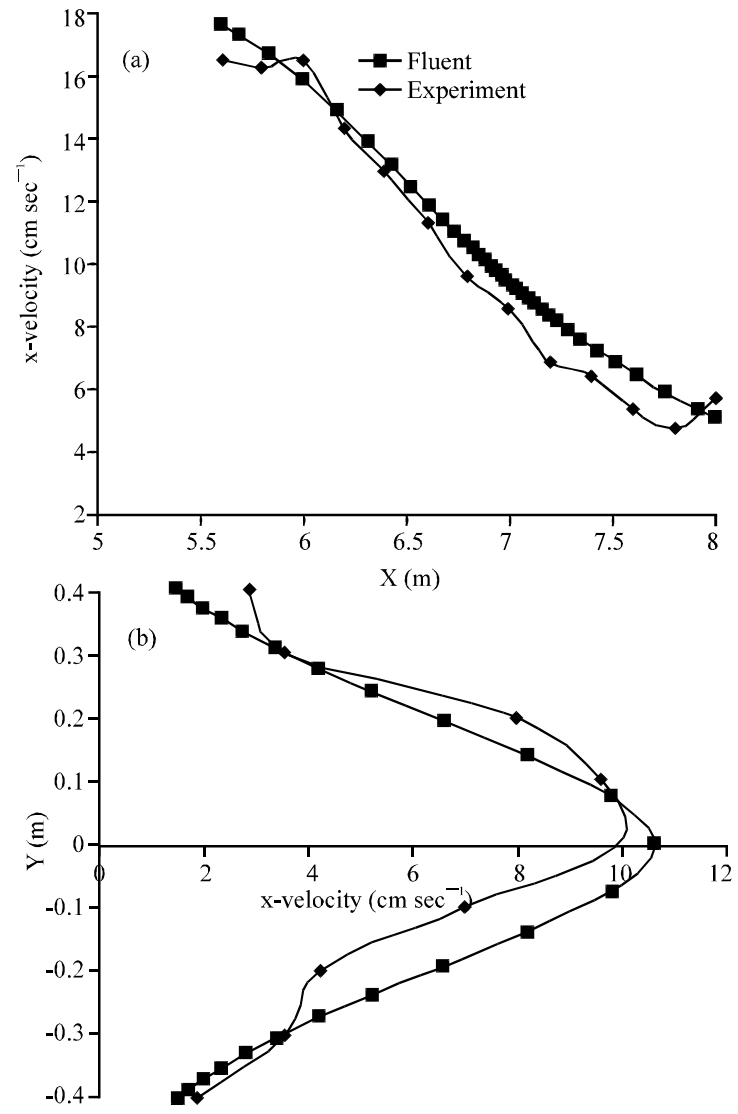

Fig. 10: $\mathrm{x}$-velocity in $11.5^{\circ}$ angle flume 

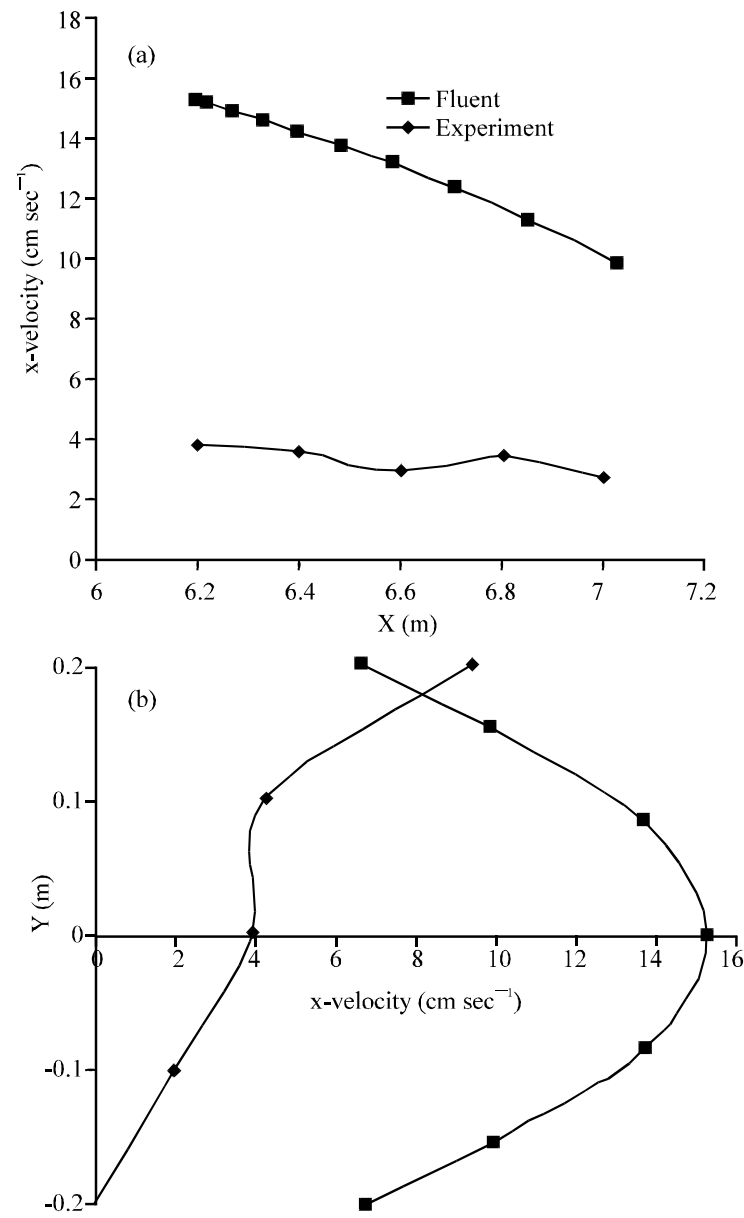

Fig. 11: X-velocity in $23.5^{\circ}$ angle flume

both is symmetric. But laboratory results in the $11.5^{\circ}$ angle are almost symmetrical and $23.5^{\circ}$ is completely asymmetric. The velocity magnitude value using the experimental results in the 23.5 angle is much less than its value using the numerical model. In $11.5^{\circ}$ angle, asymmetry length and width of the flow pattern in the flume was minor. Therefore, numerical model and experimental results are in good agreement for this angle but the $23.5^{\circ}$ angle is similar to sudden expansion and flow condition is asymmetric.

\section{CONCLUSION}

In this research, flow pattern in the sudden expansion are investigated. In this condition for medium and fine mesh size the flow pattern was symmetrical. In the case of coarse mesh size, increasing the number of iterations cause the instability of flow and symmetric flow pattern. Also, the flow pattern in a laboratory flume with sudden and gradual expansion angle is studied. Symmetric flow pattern is obtained in these conditions. Finally for study the effect of roughness on the flow pattern, the wall roughness of flume is changed and the asymmetry flow observed in this case. So, this result showed that asymmetric flow pattern could be observed due to minor changes in flume.

\section{REFERENCES}

Aloui, F. and M. Souhar, 2000. Experimental study of turbulent asymmetric flow in a flat duct symmetric sudden expansion. J. Fluids Eng., 122: 174-177.

Cherdron, W., F. Durst and J.H. Whitelaw, 1978. Asymmetric flows and instabilities in symmetric ducts with sudden expansions. J. Fluid Mech., 84: 13-31.

Escudier, M.P., P.J. Oliveira and R.J. Poole, 2002. Turbulent flow through a plane sudden expansion of modest aspect ratio. J. Phys. Fluids., 14: 3641-3654.

Graber, S.D., 1982. Asymmetric flow in symmetric expansions. J. Hydraul. Divisions, 108: 1082-1101.

Graber, S.D., 2006. Asymmetric flow in symmetric supercritical expansions. J. Hydraul. Eng., 132: 207-213.

Roul, M.K. and S.K. Dash, 2011. Two-phase pressure drop caused by sudden flow area contraction/ expansion in small circular pipes. Int. J. Numer. Methods Fluids, 66: 1420-1446.

Roy, V., S. Majumder and D. Sanyal, 2010. Analysis of the turbulent fluid flow in an axi-symmetric sudden expansion. Int. J. Eng. Sci. Technol., 2: 1569-1574.

Zhou, J.G., 1995. Velocity-depth coupling in shallow-water flows. J. Hydraul. Eng., 121: 717-724. 